\title{
Renal Aspects and Enzyme Replacement Therapy of Fabry Disease
}

\author{
Ane Cláudia Fernandes Nunes¹, Elvino José Guardão Barros², \\ Virgílio Pimentel Delgado ${ }^{3}$ and Alvimar Gonçalves Delgado 4 \\ ${ }^{1}$ Laboratory of Cellular, Genetic and Molecular Nephrology (LIM-29) \\ Division of Nephrology, São Paulo University Medical School \\ University of São Paulo, São Paulo/ \\ ${ }^{2}$ Service of Nephrology, Clinical Hospital of Porto Alegre \\ Federal University of Rio Grande do Sul, Porto Alegre \\ ${ }^{3}$ Service of Nephrology, Clementino Fraga Filho Hospital \\ Federal University of Rio de Janeiro, Rio de Janeiro \\ Federal Hospital of Bonsucesso, Rio de Janeiro \\ ${ }^{4}$ Service of Nephrology, Clementino Fraga Filho Hospital \\ Federal University of Rio de Janeiro, Rio de Janeiro \\ Brazil
}

\section{Introduction}

Fabry disease (FD) is a lysosomal storage disorder caused by the deficient $\alpha$-galactosidase A ( $\alpha$-gal A) activity. Fabry nephropathy typically progresses throughout the fifth decade to end-stage renal disease (ESRD) requiring hemodialysis and/or kidney transplantation. Except for ESRD development, a milder phenotype "renal variant" type is characterized with low plasma $\alpha$-gal A activity. This survey improved the interest for FD screening among ESRD patients. After the description of this "renal variant" of FD, many studies were designed for ESRD patients.

The prevalence of $0.24-0.36 \%$ found in Brazil express the importance of Fabry disease investigation among ESRD patients without known cause. Routine screening of male hemodialysis patients would enable earlier identification of other family members who might benefit from specific clinical treatment. The analysis of other epidemiological characteristics of regular FD could be used for the screening and detection of other kindred who might benefit from specific therapy as well as their offspring.

\section{General aspects}

Like most rare diseases, Fabry disease has long been a mystery disease, typically afflicting men of all ages and ethnic groups. With a poorly understood etiology and a disease affecting all major organ systems, little treatment other than symptomatic management has been available until recently. A patient may see many different specialists over a period of 5 to 10 years before an accurate diagnosis takes place. 
Beginning in childhood, common symptoms include chronic or intermittent numbness; burning, tingling pain that can occur daily, usually in the fingers and feet; episodic pain that is incapacitating and may be brought on by stress, exercise, or temperature changes; recurring fever with elevated erythrocyte sedimentation rate; angiokeratomas that may appear in adolescence and increase as an adult; opacity of the corneal lens; inability to perspire; severe abdominal pain; and an intolerance to temperature (heat or cold) and exercise. The condition then progresses in adulthood to include renal, cardiovascular, cerebrovascular, and pulmonary complications that may lead to end-stage renal disease, stroke, myocardial infarction, breathing problems and obstructions, and more.

In this review, we intend to explain the major aspects of Fabry disease and comment some points relatives to the characteristic of progressive renal failure in these patients.

\section{Epidemiological data}

Fabry disease is a rare inborn error with a recessive $\mathrm{X}$-linkage inherited pattern (Desnick et al., 2001). The estimated FD incidence is between 1:40,000 and 1:117,000 in general population (Desnick et al., 2003; Rolfs et al., 2005). Previous reports about the prevalence of end stage FD males on dialysis was estimated between $0.22 \%$ and $1.2 \%$ in several populations (Nakao et al., 2003; Linthorst et al., 2003; Kotamko et al., 2004; Thadhani et al., 2002; Grünfeld et al., 2003; Mehta et al., 2009).

Three similar studies explain the prevalence of FD among end-stage renal disease (ESRD) males in Brazilian population (Delgado et al., 2007; Biagini et al., 2007; Porsch et al., 2008). All studies screened about $30-40 \%$ of total patients who were submitted to dialysis treatment in three different Brazilian States: Rio de Janeiro (RJ), Paraná (PR) and Rio Grande do Sul (RS). Both first and second studies (RJ and PR) observed the same FD prevalence $(0.24 \%)$ and the third study (RS) observed a comparable value $(0.36 \%)$. FD prevalence in all studies was small compared with the results obtained by Nakao and colleagues (1.2\%) (Nakao et al., 2003) and here it is important to note that many others studies never showed the same prevalence among hemodialysis patients. In fact, even other study in Japanese population found a small prevalence $(0.22 \%)$ of FD among male dialysis patients (Ichinose et al., 2005). In addition, according to the nationwide screening for FD among dialysis patients performed in Austria (Kotamko et al., 2004), FD prevalence was really low (0.161\%). In Latin American others studies also described lower FD prevalence, akin to Peru (0.3\%) (Tumialán et al., 2005) and Colombia (0.4\%) (Martínez et al., 2005). These results support the idea that true FD prevalence is in average less than $0.5 \%$ among ESRD male and the overestimation in some studies may be related to small populations groups and/or a selection bias of the patients screened. Further studies are required in different ethnical groups with greater samples, especially among ESRD males with unknown cause for chronic renal failure.

\section{Genetic and biochemical outlines}

The enzymatic defect in FD results from the deficient activity of the $\alpha$-galactosidase A ( $\alpha$-gal A), a lysosomal hydrolase encoded by a gene (GLA) localized to Xq22. The GLA gene is 12 $\mathrm{kb}$ long and consists of 7 exons encoding 429 amino acids including a 31-amino acid signal peptide. The mature form of $\alpha$-gal A is a homodimeric glycoprotein with molecular weight of $\sim 46 \mathrm{kDa}$ synthesized from that point on cleavage of the signal peptides with $\sim 50 \mathrm{kDa}$ (Bernstein et al., 1989).

The primary substrate of this enzyme is globotriaosylceramide (galactosyl $\mathrm{l}_{\alpha 1} \rightarrow 4$ galactosyl $_{\beta 1}$ $\rightarrow 4$ glucosyl $_{\beta 1} \rightarrow 1^{\prime}$ 'ceramide), and the failure of $\alpha$-gal A activity increase the deposition of 
glycosphingolipids with terminal $\alpha$-linked galatocsyl moieties (Desnick et al., 2003). In FD this leads to progressive intracellular accumulation of glycosphingolipids, mainly in the form of globotriaosylceramide (Gb-3), in many cells, particularly in renal epithelial cells, endothelial cells, pericytes, vascular smooth muscle cells, cardiomyocytes, and neurons of the autonomic nervous system (Desnick et al., 2001). The Figure resume the cleavage events in Gb-3 to Gb-2 transition.

The genetic defect occurs in all cell types, but involvement differs greatly among different organs and cell types. This heterogeneity likely reflects different rates of sphingolipid metabolism. Thus the minimum threshold requirement for $\alpha$-gal A activity to prevent Gb-3 accumulation varies across cell types due to the type and amount of substrates that are recycled by the different cells (Alroy et al., 2002).

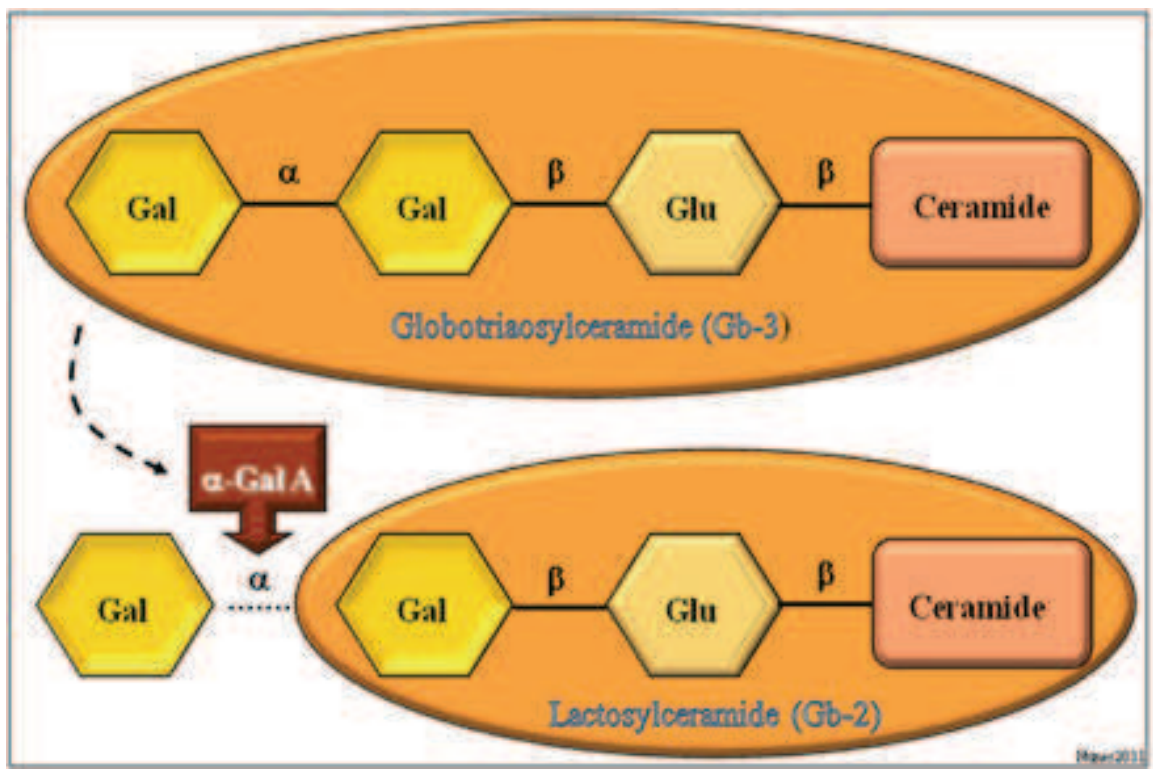

Fig. 1. Schematic representation of $\alpha$-gal A action in Gb-3 to Gb-2 cleavage into lysosomal.

Renal lesions are found in both hemizygous (male) and heterozygous (female) patients. Renal symptoms in the latter are typically milder and delayed by 2 to 3 decades, but there is considerable variability (Gubler et al., 1978). Although the disease primarily affects men, the genetic mechanism responsible allows the defect to be passed on by women. However, as women were presumed to be protected to the effects of the disease, this disorder has not been studied as comprehensively in them. The variability is likely the result of the random nature of $X$ inactivation, resulting in considerable variability in $\alpha$-gal A activity among carriers and within one carrier individual among various tissues or regions of a single tissue.

\section{Clinical diagnosis markers}

Clinical onset of the disease typically occurs during childhood or adolescence with recurrent episodes of severe pain in the extremities, characteristic cutaneous lesions know as angiokeratomas and a distinctive but asymptomatic corneal dystrophy (Clarke et al., 1971). 
Proteinuria and chronic renal disease occur with increasing age. Severe renal impairment leads to hypertension and uremia. Without dialysis, transplantation or enzyme replacement therapy (ERT), progressive renal failure is the main cause of death in the $4^{\text {th }}$ decade of life in most hemizygous males with FD. However, a number of variants with residual $\alpha$-gal A activity with late-onset manifestations primarily limited to the heart or kidney have been described (Desnick et al., 2001; Nakao et al., 1995; Meroni et al., 1997).

The 'classical phenotype' includes the pain and paresthesias in extremities, diffused angiokeratoma and hypohidrosis during childhood or adolescence, and also corneal opacities and renal failure (Clarke et al., 1971; Desnick et al., 2004). Fabry nephropathy typically progresses throughout the fifth decade of life to ESRD requiring hemodialysis and/or kidney transplantation. In view of this fact, hemodialysis patients represent an important target group for FD screening (Desnick et al., 2003; Nakao et al., 1995). Death usually occurs due to renal failure, cardiac or cerebrovascular disease. In addition, milder variants with residual $\alpha$-gal A activity have been described (Desnick et al., 2004; Nakao et al., 1995). The cardiac and renal variants present with either late-onset manifestations primarily limited to the heart or kidney (Desnick et al., 2001; Meroni et al., 1997).

Since the description of the 'renal variant' (Nakao et al., 2003), a milder FD phenotype with either late-onset manifestations primarily limited to the kidney, important dialysis screening efforts of ESRD populations have been carried out (Nakao et al., 2003; Linthorst et al., 2003; Torra et al., 2003; Kotamko et al., 2004; Thadhani et al., 2002) and seemed to be worthwhile since kidney failure is an important outcome in FD.

While in an epidemiological point of view FD occurrence is low, on the other hand the FD diagnosis is very important for detection of family members. In view of this fact, dialysis patients represent an important target group for FD screening because they permit to identify FD patients and therefore others carriers among your family members. Each screened confirmed patient could allow early diagnosis of others related subjects, who can get treatment before or in the earlier symptoms manifestations. In these terms, FD screening among ESRD patients consists of an important tool for detection of FD patients and it could be followed by FD screening between family members of the index case. Both pedigree and population screening studies have been described and it can be carried out in subpopulations thought to be at higher risk of disease than the general population (Warnock et al., 2005).

\section{Guess of renal damage}

There are some references that the renal Gb-3 content, renal pathology and renal function correlate with residual $\alpha$-gal A activity in leukocytes (Branton et al., 2002). If renal $\alpha$-gal A activity correlates with leukocyte $\alpha$-gal A activity (a reasonable but untested assumption), this suggests that residual enzyme activity in renal parenchymal cells retards progression of renal disease. One case report has suggested that renal $\alpha$-gal A activity was reduced compared with liver $\alpha$-gal A activity when each was expressed as a fraction of normal $\alpha$-gal A activity in that organ; the mechanism for such a finding is unclear (Kano et al., 1974). Similarly, were found that Fabry patients with conservative missense mutations have delayed appearance of renal disease compared with patients with nonconservative missense mutations or others mutations those resulting in deletions, insertions, or premature stop codons (Branton et al., 2002). 
According Alroy and colleagues (Alroy et al., 2002) three mechanisms might explain the segmental and global glomerulosclerosis that characterizes Fabry disease: microvascular disease, podoctyte injury, and tubulointerstitial injury. Gubler and colleagues (Gubler et al., 1978) observed that in older Fabry patients, those 25 to 50 year old, the progressive renal pathologic changes in the glomeruli and tubulointerstitium may be related to ischemic change. These changes include glomerulosclerosis, often with wrinkled and partially collapsed glomerular basement membrane, tubular atrophy, interstitial fibrosis, and vascular thickening. These changes were generally absent or mild in patients under 25 year of age. In particular, these investigators noted that the earliest and most consistent degenerative alteration was arterial "fibrinoid" deposits and suggested that these were due to necrosis of smooth muscle cells fatally overloaded with Gb-3 deposits.

Hypertension is not a common feature of Fabry disease, although it may occur with progressive renal dysfunction (Branton et al., 2002). Therefore, according Gubler and colleagues (Gubler et al., 1978), one mechanism of renal injury in Fabry disease is accumulation of Gb-3 within the arterial vessel wall and subsequent vascular compromise. In this regard, the renal vasculature is similar to the coronary and cerebral vessels, in which large vessel deposition of Gb-3 is associated with premature vascular disease that is responsible for premature death in many patients.

Toxic accumulation of Gb-3 within the podocyte may constitute a second important mechanism of glomerular injury. Podocytes are highly differentiated cells; their foot processes and slit-diaphragms constitute a critical portion of the glomerular filtration barrier that retards the entry large molecules into the urinary space. These cells are post mitotic and fail to undergo proliferation under most pathologic circumstances (an exception being the collapsing variant of focal segmental glomerulosclerosis), which means that they generally are not replaced when they are lost due to lethal injury. Kriz and Lemley (Kriz \& Lemlev, 1999) have proposed that when podocytes are lost, the denuded glomerular basement contacts the parietal epithelial cells and forms a synechia. Within the synechia, there is activation and proliferation of cells, especially mesangial cells, the entry of immune cells, including macrophages, and the accumulation extracellular matrix protein. This repair response may be driven in part by the leakage from the circulation into the synechia of macromolecules, including cytokines, chemokines, and growth factors, via the impaired glomerular filtration barrier. Matrix expansion and subsequent collapse of the capillary loop appears as the focus of solidification. This constitutes the lesion of segmental glomerulosclerosis, which progresses to global glomerulosclerosis.

The Gb-3 also induces podocyte injury, resulting in focal and ultimately global glomerulosclerosis. Deposition of Gb-3 within tubular epithelial cells may lead to focal tubular atrophy and interstitial fibrosis. As this process progresses, the glomeruli upstream of more severely affected tubules may function poorly or not at all. Other glomeruli may undergo hypertrophy to compensate, and hyperfiltration in these glomeruli may trigger a secondary form of focal segmental glomerulosclerosis. Evidence in support of this mechanism would include demonstration of glomerular enlargement, particularly in the early stages of glomerular and tubular injury in Fabry disease.

Alternatively, Eng and colleagues (Eng et al., 2001a) proposed the change in microvascular inclusions in interstitial endothelial cells of kidney, heart, and skin as a primary endpoint in their recent trial of $\alpha$-gal A replacement. But the importance of this parameter as a potential substitute marker for the progression of renal dysfunction (impaired GFR) and other renal pathology (glomerulosclerosis, interstitial fibrosis) is uncertain and needs to be tested in 
longitudinal studies. Some additional pathologic markers, as mesangial expansion, glomerulosclerosis, and interstitial fibrosis were included in another trial of $\alpha$-gal A replacement (Schiffmann et al., 2000), although these pathologic markers are also untested as appropriate markers for progressive renal functional decline in Fabry disease.

\section{Urinary concentration and proteinuria changes}

Urinary concentration defects may be the earliest functional manifestation of Fabry renal disease, leading to polyuria and nocturia. However, nephrology referral is more typically initiated by the development of proteinuria. Proteinuria may begin in the teenage years and becomes more frequent when patients reach their 20s and 30s.

In the NIH series (Branton et al., 2002), 33 of 34 patients who had urine protein electrophoresis were found to have glomerular proteinuria, although the proteinuria did not usually reach nephrotic levels. Indeed, $23 \%$ of patients progressed to chronic renal insufficiency (CRI) without ever having nephrotic proteinúria and $50 \%$ of patients developed CRI by 43 year of age. The full presentation of nephrotic syndrome was not frequent even in those patients who developed nephrotic-range proteinuria. Only $26 \%$ of patients with nephrotic-range proteinuria developed hypoalbuminemia, and $21 \%$ developed hyperlipidemia. The onset of CRI may begin as early as the second decade of life. The mean age of onset of clinical nephropathy (CRI or proteinuria) has been reported as 27 year (Donati et al., 1987).

End-stage renal disease was the most common cause of death in Fabry patients before the development of dialysis and renal transplantation and ESRD may rarely occur during the teenage years (Branton et al., 2002).

Progression from onset of CRI to ESRD occur in mean of $4 \pm 3 \mathrm{yr}$ (range, 1 to $13 \mathrm{yr}$ ) and was not affected by patient age at onset of CRI or magnitude of proteinuria (Branton et al., 2002). Besides that, patients with undetectable residual $\alpha$-gal A activity had higher scores for glomerular pathology $(P=0.027)$ and tubulointerstitial pathology $(P=0.007)$, and they also had higher concentrations of Gb-3 in kidney tissue $(P=0.039)$ (Branton et al., 2002).

\section{Improve in treatment in recent years}

Fabry patients with proteinuria or CRI should have aggressive treatment of hypertension is present and should probably be treated preferentially with angiotensin antagonist therapy; the latter recommendation is based on theoretical considerations, as definitely proof of efficacy has not been obtained yet.

Two different recombination $\alpha$-gal A preparations are in use for treating Fabry disease (Schiffmann et al., 2001a; Eng et al., 2001a). One enzyme is produced by Chinese hamster ovary $(\mathrm{CHO})$ cells with classic recombinant technology (agalsidase $\beta$, Fabrazyme - Genzyme Corporation), and the other enzyme is produced by cultured human skin fibroblast with an activated promoter of the $\alpha$-gal A gene (Agalsidase $\alpha$, Replagal - Shire Human Genetics Therapies). Both recombinant enzymes are quite comparable in properties and differ only alightly in glycan composition (Blom et al., 2003). The two enzyme preparations have independently been examined in clinical investigations and are both registered in Europe for treating Fabry patients. Although both enzyme therapies were found to result in the desired Gb-3 from endothelium, the clinical effects are not robust as anticipated. In some 
patients, stabilization of renal function and improvenment in cardiac hypertrophy occurs upon therapy, but a considerable number experiences progressive complications (Vedder et al., 2007)

The NIH carried out a double-blind, randomized, placebo-controlled study of recombinant $\alpha$-gal A produced in a human cell line (Replagal; Shire Human Therapies) and administered by biweekly infusion to 26 male Fabry patients for 6 months (Schiffmann et al., 2001a). The primary endpoint of this study was a reduction in neuropathic pain. Significant reductions in the severity of "pain at its worst" and in the use of chronic pain medications were demonstrated. Enzyme replacement therapy was associated with relatively few side effects, chiefly mild transient infusion reactions, which became uncommon when the infusion duration was increased (from 20 to $40 \mathrm{~min}$ ).

In the same study of Schifmann and colleagues, histologic assessment of renal biopsy samples showed that enzyme therapy was associated with more normal glomeruli $(P<0.01)$ and fewer glomeruli exhibiting mesangial matrix widening $(P<0.01)$, as well a slight increase in segmentally sclerotic glomeruli $(P<0.05)$ and no change in the number of globally sclerotic glomeruli. There were relatively few globally sclerotic glomeruli; therefore, the overall effect was an improvement in glomerular architecture. There was no change in the composite scores for tubulointerstitial damage or glycolipid deposits (the latter was assessed on toluidine blue- stained sections as the sum of scores in podocytes, glomerular endothelial/mesangial cells, proximal tubular epithelial cells, distal tubular epithelial cells, extraglomerular vascular endothelial cells, and vascular medial cells). Interestingly, there was however a significant decrease in glycolipid deposits in vascular endothelial cells $(P<$ 0.002). Enzyme replacement therapy was associated with a significant fall in Gb-3 concentrations in plasma and urine sediment, but the findings in kidney tissue biopsies were not significant (NS) (Schiffmann et al., 2001a). Six months of enzyme therapy had no consistent effect on proteinuria. With respect to the effect of enzyme replacement therapy on renal function, there was a trend toward a greater fall in GFR, measured by inulin clearance, with placebo compared with enzyme therapy $(P=0.27)$ (Schiffmann et al., 2001a). Thus, this results was encouraging but insufficient to demonstrate a definitive benefit of ERT with $\alpha$ gal A on kidney function in the group studied during a small period of treatment.

In another study, Thofhern and colleagues (Thofhern et al., 2009), available 9 patients (7 male, 2 female) during the period between January 1, 2002, and August 1, 2005. They were treated according to protocol, receiving $0.2 \mathrm{mg} / \mathrm{kg}$ agalsidase alfa IV every two weeks. Over the course of 36 months of ERT, there was no change in kidney function and 24-hour proteinuria. This suggests that agalsidase alfa may slow or halt the progression of kidney disease when used before extensive kidney damage occurs. No significant side effects were observed with ERT during the course of this study.

Recent results from an open-label extension of enzyme replacement in patients enrolled in the original trial of NIH showed that patients in the initial treatment group continued to have stable or improved renal function measured by inulin and creatinine clearance after 8 months of enzyme therapy. In contrast, patients originally in the placebo group who had shown a decline in renal function demonstrated significantly improved inulin clearance and creatinine clearance after 12 months of enzyme replacement therapy (Schiffmann et al., 2001b). Improved intracardiac conduction as evidenced by significant reduction of QRS complex duration in electrocardiogram (ECG) after 6 months of enzyme treatment was also noted. These new results with enzyme replacement are encouraging and results of continued therapy will be interesting to follow. 
Eng and colleagues (Eng et al., 2001b) evaluated the efficacy of recombinant human a-gal A produced in Chinese hamster ovary cell line (Fabrazyme; Genzyme, Boston, MA) in Fabry patients. In an open-label, doseranging study involving 15 patients, these authors found that biweekly infusions given for 10 weeks was associated with reduced kidney Gb-3 content (5 of the 15 patients underwent paired renal biopsies; no statistics given). On glutaraldehydefixed, methylene blue-stained kidney biopsy tissue, there was reduced storage material in interstitial capillary endothelial cells and mesangial cells. There was a lesser degree of improvement or no improvement in tubular epithelial cells and glomerular podocytes.

These results obtained by Eng and colleagues were used to design The International Fabry Disease Study, a double blind, randomized placebo controlled trial involving 58 male Fabry patients treated with biweekly intravenous infusions for 20 weeks, with the primary endpoint being clearance of interstitial capillary endothelial cell deposits (Eng et al., 2001a). Complete clearance of interstitial capillary endothelial cell deposits occurred in 20 of 29 of $\alpha$ gal A-treated patients and 0 of 29 placebo-treated patients $(P<0.001)$. Similar significant changes in endothelial cell deposits were seen in skin and heart capillaries. There was no beneficial effect on GFR assessed by inulin clearance after 20 weeks of ERT. There was a transient improvement in pain scores that did not persist to week 20, but pain results were no different than with placebo.

However, after 11 months of additional open label treatment with agalsidase beta, the renal effects of this ERT were more completely evaluated: clearance of Gb-3 from glomerular endothelial cells, clearance from smooth muscle cells, mesangial cells and cortical interstitial cells, and some clearance from podocytes (Thurberg et al., 2002). These results showing a clear renal benefit with the ERT was followed by an extension for an additional 54 months of treatment with agalsidase beta: complete clearance from endotelial and other cells was maintained and renal function remained stable in all but six patients, in wich progressive disease was associated with significant baseline proteinuria and more glomerular sclerosis (Germain et al., 2007). After that, agalsidase beta (Fabrazyme) was approved for marketing in Europe in 2001, and in the United States in 2003 with the main indication for the reduction in the accumulation of Gb-3 deposits in the capillary endothelium of the kidney. Agalsidase alfa (Replagal) is approved for marketing in Europe and many other countries. Both was recently approved for use in Brazil.

Data from the phase IV trial with agalsidase beta in 82 adults with Fabry disease and renal dysfunction at baseline (creatinine concentration between 1.2 and 3.0 or estimated creatinine clearance less than $80 \mathrm{~mL} / \mathrm{min}$ ), randomly assigned in a two to one treatment to placebo ratio during 35 months, showed a trend to better clinical effect with treatment, although no statistically significant (Banikazemi et al., 2007). Importantly, a secondary analysis found benefits with therapy in those patients with renal function relatively well preserved (clearance $\geq 55 \mathrm{~mL} / \mathrm{min} / 1.73 \mathrm{~m}^{2}$ ) for those with renal function less than $55 \mathrm{~mL} / \mathrm{min} / 1.73 \mathrm{~m}^{2}$. In addition, an open label treating 58 patients with $1 \mathrm{mg} / \mathrm{kg}$ of agalsidase beta for 30 to 36 months demonstrated that baseline proteinuria less than $1 \mathrm{~g}$ and glomeruloesclerosis less than $50 \%$ showed better evolution and better renal prognostic (Wilcox et al., 2004). All these data suggest that starting therapy before extensive renal damage has been identified might be beneficial for the prevention of progression to renal failure.

In summary, some placebo-controlled clinical trials have shown that 6 months of enzyme replacement therapy with $\alpha$-gal $\mathrm{A}$ is associated with improved glomerular architecture and/or reduced glycolipid deposits in the kidney, and one study also suggested improvement in renal function. These trials have recently been reviewed (Pastores et al., 
2002). It makes sense that vascular endothelial cells are especially responsive to intravenously administered enzyme, in that these cells have direct access to the $\alpha$-gal A present in the circulation. The majority of Gb-3 deposition in the kidney, however, does not appear to be within these cells. Indeed, Schiffmann and colleagues also demonstrated improved glomerular architecture, and results of follow-up studies of renal function are forthcoming (Schiffmann et al., 2001a/b).

All the results of International Study of Fabry Disease with agalsidase beta, including double blind studies, open-label studies and extension studies has shown the best evidence of benefit on the renal function, with low rate of adverse events. Data of these studies demonstrated that $1 \mathrm{mg} / \mathrm{kg}$ each other week of enzyme replacement are capable of stabilize the renal function mainly when the clearance is relatively well preserved $(\geq 55$ $\mathrm{mL} / \mathrm{min} / 1.73 \mathrm{~m}^{2}$ ), the glomeruli and possibly interstitial tissue is not extensive damaged and the proteinuria is less than $1 \mathrm{~g}$ per day.

Additional studies as to whether $\alpha$-gal A therapy can prevent, slow, halt, or reverse declining renal function in patients with Fabry disease will likely be of 1 year or longer duration, possibly selecting only patients with renal insufficiency for inclusion to maximize the chance of showing a benefit in renal function. Nevertheless, at the present time enzyme replacement therapy holds considerable promise for patients with Fabry disease with and without kidney involvement.

\section{Final considerations}

As treatment for Fabry disease is now available the enzyme replacement therapy. This treatment, among other benefits, reduced Gb-3 depositions in renal tissue and could represent a better outcome for FD patients. However, this approach is important if applied before the onset of ESRD when the efficiency of treatment is more evidenced.

This fact also indicates the importance of FD diagnosis before the classical symptoms appear. A better awareness about FD among physicians and scientists should increase the number of patients that will be identified by different specialists and through population and family screening. Subsequently, FD diagnosis should be considered in every patient with unexplained renal disease, especially in patients with painful uncleared episodes, disseminate cutaneous lesions or when cardiac or cerebral complications suggest an underlying mutisystemic disorder.

The results presented here stresses the importance of Fabry disease reports since a single FD diagnosed patient by screening is a potential indicator for finding others Fabry carriers within his family. Because enzyme replacement therapy by recombinant $\alpha$-gal A has emerged as a promising means to prevent and remove Gb-3 deposition, it is now necessary to make this diagnosis earlier.

\section{References}

Alroy J, Sabnis S, Kopp JB. (2002). Renal Pathology in Fabry disease. J Am Soc Nephrol 13: S134-138.

Banikazemi M, Bultas J, Waldek S, et al. (2007) Agalsidase-beta therapy for advanced Fabry disease: a randomized trial. Ann Intern Med; 146:77.

Biagini G, Pecoits RRPF and Weber HHMW. (2007) Prevalence of Fabry disease in patients with chronic renal insufficiency undergoing hemodialysis in the State of Paraná - 
Brazil. Book of Abstracts (pp356). World Congress of Nephrology, Rio de Janeiro, Brazil.

Blom D, Speijer D, Linthorst GE, et al. (2003) Recombinant enzyme therapy for Fabry disease: absence of editing of human alpha-galactosidase A mRNA. Am J Hum Genet 72: 23-31.

Chamoles NA, Blanco M, Gaggioli D. (2001) Fabry disease: enzymatic diagnosis in dried blood spots on filter paper. Clin Chim Acta 308(1-2): 195-6.

Clarke JT, Knaack J, Crawhall JC, Wolfe LS. (1971) Ceramide trihexosidosis (Fabry's disease) without skin lesions. N Engl J Med. 284(5):233-5.

Delgado V, Nunes ACF, Canuto M, et al. (2007) Fabry disease epidemiology in Rio de Janeiro State: Partial outcome of screening in patients on dialysis treatment. Book of Abstracts (pp280). World Congress of Nephrology, Rio de Janeiro, Brazil.

Desnick R, Ioannou Y, Eng C. (2001) $\alpha$-galactosidase a deficiency: Fabry disease. In: Scriver CR, Sly WS, Beaudet AL, Valle D, editors. The metabolic and molecular bases of inherited disease. 8th ed. New York: McGraw-Hill. pp. 3733-4.

Desnick R.J., Brady R, Barranger J, et al. (2003) Fabry disease, an under-recognized multisystemic disorder: expert recommendations for diagnosis, management, and enzyme replacement therapy. Ann Intern Med 138(4): p. 338-46.

Desnick R.J. and Brady R.O. (2004) Fabry disease in childhood. J Pediatr 144(5 Suppl): p. S206.

Donati D, Novario R, Gastaldi L: (1987) Natural history and treatment of uremia secondary to Fabry's disease: A European experience. Nephron 46: 353-359.

Eng CM, Guffon N, Wilcox WR, et al. (2001a) Safety and efficacy of recombinant human alpha-galactosidase A-replacement therapy in Fabry's disease. N Engl J Med 345: 916.

Eng CM, Banikazemi M, Gordon RE, et al. (2001b) A phase 1/2 clinical trial of enzyme replacement in fabry disease: Pharmacokinetic, substrate clearance, and safety studies. Am J Hum Genet 68: 711-722.

Germain DP, Waldek S, Banikazemi M, et al. (2007) Sustained, long-term renal stabilization after 54 months of agalsidase beta therapy in patients with Fabry disease. J Am Soc Nephrol 18:1547.

Grünfeld J-P. (2003) How to improve the early diagnosis of Fabry disease? Kidney Int 64: 1136-7.

Gubler MC, Lenoir G, Grunfeld JP, et al. (1978) Early renal changes in hemizygous and heterozygous patients with Fabry's disease. Kidney Int 13: 223-235.

Ichinose M, Nakayama M, Ohashi T. et al. (2005) Significance of screening for Fabry disease among male dialysis patients. Clin Exp Nephrol 9: 228-32.

Kotamko P, K.R., Kramar R, Devrnja D et al. (2004) Results of a nationwide screening for Anderson-Fabry disease among dialysis patients. J Am Soc Nephrol 15: p. 1323-1329.

Kriz W and Lemley KV. (1999) The role of the podocyte in glomerulosclerosis. Curr Opin Nephrol Hypertens 8: 489-497.

Linthorst GE, G., Hollak CE, Korevaar JC et al. (2003) Alpha-Galactosidase A deficiency in Dutch patients on dialysis: a critical appraisal of screening for Fabry disease. Nephrol Dial Transplant 18:1581-1584. 
Martínez JA, Ardila ME, Gamarra G, et al. (2005) Prevalence of Fabry disease in Colombia Preliminary report. Book of Abstracts (pp237). X Latin American Symposium in Lysosomal Storage Disease, San Jose, Costa Rica.

Meroni M, Sessa A, Battini G, et al. (1997) Kidney involvement in Anderson-Fabry disease. Contrib Nephrol 122:178-84.

Mehta A, Beck M, Elliot P. Enzyme replacement therapy with agalsidase alfa in patients with Fabry's disease: an analysis of registry data. Lancet 374(9706): 1986-96.

Morgan SH, Rudge P, Smith SJ, et al. (1990) The neurological complications of AndersonFabry disease (alpha-galactosidase A deficiency): investigation of symptomatic and presymptomatic patients. Q J Med 75: 491-507.

Nakao S, T.T., Maeda M et al. (1995) An atypical variant of Fabry disease in men with left ventricular hypertrophy. N. Engl. J. Med.333: 288-293.

Nakao S, Kodama C, Takenaka T, et al. (2003) Fabry disease: detection of undiagnosed hemodialysis patients and identification of a "renal variant" phenotype. Kidney Int 64(3):801-7.

Nunes ACF, Delgado V, Porsch DB, et al. (2007) Fabry disease and hemodialysis: Determining a procedure for blood collecting on a Brazilian screening. Book of Abstracts (pp279). World Congress of Nephrology, Rio de Janeiro, Brazil.

Pastores GM and Thadhani R. (2002) Advances in the management of Anderson-Fabry disease: Enzyme replacement therapy. Expert Opin Biol Ther 2: 325-333.

Porsch DB, Nunes ACF, Milani V, et al. (2008) Fabry Disease in Hemodialysis Patients in Southern Brazil: Prevalence Study and Clinical Report. Renal Failure 30:825-30.

Ries M, Ramaswami U, Parini R, et al. (2003) The early clinical phenotype of Fabry disease: a study on 35 European children and adolescents.Eur J Pediatr 162: 767-772.

Rolfs A, B.T., Zschiesche M et al. (2005) Prevalence of Fabry disease in patients with cryptogenic stroke: a prospective study. Lancet 366:1794-1796.

Sachdev B, Takenaka T, Teraguchi T et al., (2002) Prevalence of Anderson-Fabry disease in male patients with late onset hypertrophic cardiomyopathy. Circulation 105:14071411.

Schiffmann R, Murray GJ, Treco D, et al. (2000) Infusion of alpha-galactosidase A reduces tissue globotriaosylceramide storage in patients with Fabry disease. Proc Natl Acad Sci USA 97: 365-370.

Schiffmann R, Kopp JB, Austin HA, et al. (2001a) 3rd, Sabnis S, Moore DF, Weibel T, Balow JE, Brady RO: Enzyme replacement therapy in Fabry disease: A randomized controlled trial. JAMA 285: 2743-2749.

Schiffmann R, Kopp JB, Moore DF, et al. (2001b) Efficacy and safety of prolonged enzyme replacement therapy for Fabry disease. Am J Hum Genet 69(Suppl): 222.

Thadhani R, Wolf M, West ML, et al.(2002) Patients with Fabry disease on dialysis in the United States. Kidney Int 61(1):249-55.

Thofhern S, Netto C, Cecchin C, et al. (2009) Kidney Function and 24-Hour Proteinuria in Patients with Fabry Disease during 36 Months of Agalsidase Alfa Enzyme Replacement Therapy: A Brazilian Experience. Renal Failure 31: 773-778.

Thurberg BL, Rennke H, Colvin RB, et al. (2002) Globotriaosylceramide accumulation in the Fabry kidney is cleared from multiple cell types after enzyme replacement therapy. Kidney Int 62:1933. 
Tumialán C, Carvalho C, Chavez R., et al. (2005) Prevalence in Peru of Fabry disease among males with chronic renal insufficiency. Book of Abstracts (pp191). X Latin American Symposium in Lysosomal Storage Disease, San Jose, Costa Rica.

Warnock, D. (2005) Fabry disease: diagnosis and management, with emphasis on the renal manifestations. Cur Opinion in Nephrol and Hypert 14: p. 87-95.

Wilcox WR, Banikazemi M, Guffon N, et al. (2004) Long-term safety and efficacy of enzyme replacement therapy for Fabry disease. Am J Hum Genet 75:65.

Vedder AC, Linthorst GE, Houge G, et al. (2007)Treatment of Fabry disease: outcome of a comparative trial with agalsidase alfa or beta at a dose of $0.2 \mathrm{mg} / \mathrm{kg}$. PLOS ONE 2:e598. 


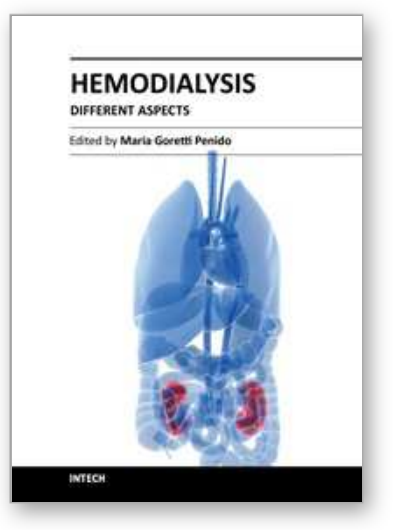

\author{
Hemodialysis - Different Aspects \\ Edited by Prof. Maria Goretti Penido
}

ISBN 978-953-307-315-6

Hard cover, 321 pages

Publisher InTech

Published online 14, November, 2011

Published in print edition November, 2011

The book provides practical and accessible information on all aspects of hemodialysis, with emphasis on dayto-day management of patients. It is quite comprehensive as it covers almost all the aspects of hemodialysis. In short it is a valuable book and an essential aid in the dialysis room.

\title{
How to reference
}

In order to correctly reference this scholarly work, feel free to copy and paste the following:

Ane Cláudia Fernandes Nunes, Elvino José Guardão Barros, Virgîlio Pimentel Delgado and Alvimar Gonçalves Delgado (2011). Renal Aspects and Enzyme Replacement Therapy of Fabry Disease, Hemodialysis - Different Aspects, Prof. Maria Goretti Penido (Ed.), ISBN: 978-953-307-315-6, InTech, Available from: http://www.intechopen.com/books/hemodialysis-different-aspects/renal-aspects-and-enzyme-replacementtherapy-of-fabry-disease

\section{INTECH}

open science | open minds

\section{InTech Europe}

University Campus STeP Ri

Slavka Krautzeka 83/A

51000 Rijeka, Croatia

Phone: +385 (51) 770447

Fax: +385 (51) 686166

www.intechopen.com

\section{InTech China}

Unit 405, Office Block, Hotel Equatorial Shanghai

No.65, Yan An Road (West), Shanghai, 200040, China

中国上海市延安西路65号上海国际贵都大饭店办公楼405单元

Phone: +86-21-62489820

Fax: +86-21-62489821 
(C) 2011 The Author(s). Licensee IntechOpen. This is an open access article distributed under the terms of the Creative Commons Attribution 3.0 License, which permits unrestricted use, distribution, and reproduction in any medium, provided the original work is properly cited. 